\title{
Diffusion-weighted MR imaging diagnostic merits in the post-therapeutic assessment of musculoskeletal soft tissue sarcoma
}

\author{
Amr Farouk Ibrahim Moustafa ${ }^{1 *}$ D, Shaimaa Shokry Mohammed Al Shahat El Said', \\ Maged Abdelrahman Moustafa ${ }^{2}$, Marwa Mahmoud Hussein ${ }^{3}$ and Ahmed Mohamed Shokry ${ }^{1}$
}

\begin{abstract}
Background: The purpose of the study is assessing the diagnostic merits of diffusion-weighted imaging (DWI) with apparent diffusion coefficient (ADC) mapping in evaluating tumor response to chemo-radiotherapy. The study included 36 patients with soft tissue sarcoma, who received chemo/radiotherapy. Tumor longest dimension according to response evaluation criteria in solid tumors 1.1 (RECIST 1.1), the longest dimension of the contrastenhanced portion of the tumor according to modified response evaluation criteria in solid tumors: (mRECIST), the tumor volume $(\mathrm{VOL})\left(\mathrm{cm}^{3}\right)$, and DWI with $A D C$ values were recorded.

Results: $A D C$ values in the non-progressive group were higher than those of the progressive group after neoadjuvant treatment $(1.63 \pm 0.42$ vs. $1.24 \pm 0.35)$ with $(p<0.005)$. ADC variations in the non-progressive group were higher than those of the progressive group $(27.09 \pm 48.09$ vs. $-3.08 \pm 23.5) \%$ with $(p<0.05)$. ADC values after neoadjuvant treatment were negatively related to tumor volume variations (VOL\%) after neoadjuvant treatment. $A D C$ variations (ADC\%) were inversely correlated with morphologic changes, regardless of the effectiveness of anticancer therapy expressed as changes in tumor size based on (RECIST, mRECIST, and three-dimensional volumetric assessment). An increase in the ADC value was not always associated with a reduction of tumor volume.

Conclusion: Quantitative DW imaging after neoadjuvant therapy provides added value in determining treatment response in soft tissue sarcomas. Therapeutic response to neoadjuvant therapy can be underestimated using RECIST 1.1; therefore, the mRECIST should also be considered.
\end{abstract}

Keywords: Soft tissue sarcomas, Diffusion-weighted imaging, ADC, RECIST 1.1, mRECIST

\section{Background}

Soft tissue sarcomas (STSs) are a gathering of uncommon malignancies that make up just $1-2 \%$ of all tumors in grown-ups while representing a higher extent of $15 \%$ of all malignancies in children. The challenges of treating STSs by far are due to the heterogeneous nature of this group of diseases [1].

The major therapeutic objective for all patients with STS is to achieve long-term survival, avoidance of recurrence, maximizing function, and minimizing morbidity [2].

\footnotetext{
* Correspondence: amrfaroukmoustafa@cu.edu.eg

${ }^{1}$ Department of Diagnostic and Interventional Radiology, National cancer institute, Cairo University, 1 kasr Elainy street Fom Elkalig, Cairo, Egypt Full list of author information is available at the end of the article
}

Careful resection (with suitably negative margins) is the standard essential treatment for most patients with STS, albeit close edges might be important to protect uninvolved critical neurovascular structures [3].

Radiotherapy and/or chemotherapy (on account of chemosensitive histologies) are often used before surgery in many centers to downstage enormous, high-grade tumors to empower compelling careful resection [3].

Preoperative radiotherapy may decrease seeding during the surgical manipulation of the tumor [4].

Chemotherapy with single or blend regimens has been widely used for patients with advanced, unresectable, or metastatic disease [5].

Magnetic resonance imaging (MRI) plays a vital role in the characterization of musculoskeletal lesions. MRI offers 
unique contributions to the determination and monitoring of the therapeutic response in STS. MRI can provide an assessment of the tumor vasculature, and has the potential to provide detailed information about the biochemical environment, tissue composition, and structure $[6,7]$.

Advanced techniques, such as diffusion-weighted imaging (DWI), are investigated and may turn into a crucial piece of tumor assessment. They do not require the use of intravenous contrast administration and can promptly be fused into a routine MR imaging protocol with minimal extra scan time [6].

Since cellular changes are relied upon to go before morphologic changes in tumor volume, it has been recommended that DWI may show proof of good treatment reaction sooner than conventional imaging [6].

The aim of the study was to evaluate the role of quantitative diffusion-weighted imaging with apparent diffusion coefficient (ADC) mapping in the evaluation of the response of STS to neoadjuvant therapy and to attempt to define the threshold or cut off value for the change in $A D C$ values to assess post-treatment response.

\section{Methods}

\section{Patients}

This retrospective study included 36 patients, 24 males and 12 females; their ages ranged from 2 to 66 years with the median age of 27 years, and mean age $28 \pm 20.4$ years.

Pathologically proven soft tissue sarcoma patients receiving neoadjuvant chemo and/or radiotherapy were referred to radiology department for assessment of response to the neoadjuvant therapy. Patients were excluded if the mass is poorly identified due to artifacts.

\section{Examinations performed}

- MRI was performed on a high field system (1.5 Tesla) closed magnet unit (Phillips Achieva XR).

- Initial baseline MRI was performed for all patients before the initiation of neoadjuvant therapy and all patients performed at least one post-treatment follow up MRI examination.

\section{MR protocol used}

All patients were submitted to the following MR sequences.

- Multiplanar MR imaging sequences without contrast including T1 and T2 WIs with fat-suppressed images utilizing $1.5 \mathrm{~T}$ scanner.

- Gadolinium-enhanced T1-weighted images were obtained in the coronal, axial, and/or sagittal plane.

Table 1 Patient demographics included in this study

\begin{tabular}{|c|c|c|c|}
\hline & Pathology & Number of cases & Percentage \\
\hline & Synovial sarcoma & 10 & $27.77 \%$ \\
\hline & Rhabomyosacroma RMS & 9 & $24.9 \%$ \\
\hline & Malignant peripheral nerve sheath tumor MPNT & 2 & $5.55 \%$ \\
\hline & Liposarcoma & 4 & $11.1 \%$ \\
\hline & Leimyosarcoma & 2 & $5.55 \%$ \\
\hline & Myofibroblastic Sarcoma & 2 & $5.55 \%$ \\
\hline & Fibrosarcoma & 2 & $5.55 \%$ \\
\hline & Undifferentiated sarcoma & 3 & $8.3 \%$ \\
\hline & Round cell tumor & 2 & $5.55 \%$ \\
\hline \multicolumn{4}{|c|}{ Post-operative pathology } \\
\hline \multirow[t]{2}{*}{7 patients } & Optimal response & Suboptimal response & $\begin{array}{l}\text { No comment on degree of necrosis } \\
\text { and tumor viability }\end{array}$ \\
\hline & 3 & 2 & 2 \\
\hline \multicolumn{4}{|c|}{ Site of the primary tumor } \\
\hline \multirow[t]{2}{*}{ Location } & Lower extremity & Upper extremity & Trunk \\
\hline & $25 / 36$ & $6 / 36$ & $2 / 36$ \\
\hline \multicolumn{4}{|c|}{ Treatment plan } \\
\hline \multirow[t]{2}{*}{ Treatment } & Chemotherapy & Concomitant chemo-radiotherapy (CCRTH) & Surgery following chemotherapy \\
\hline & 28/36 (77.7\%) & $8 / 36(22.2 \%)$ & $7 / 36(19.4 \%)$ \\
\hline \multicolumn{2}{|c|}{$\begin{array}{l}\text { The interval between initial baseline MRI study } \\
\text { and the follow-up MRI exams }\end{array}$} & \multicolumn{2}{|c|}{$\begin{array}{l}\text { Ranging from } 1 \text { month up to } 1 \text {-year post-therapy, with an average of } \\
\text { about } 4.5 \text { months duration of follow up }\end{array}$} \\
\hline
\end{tabular}



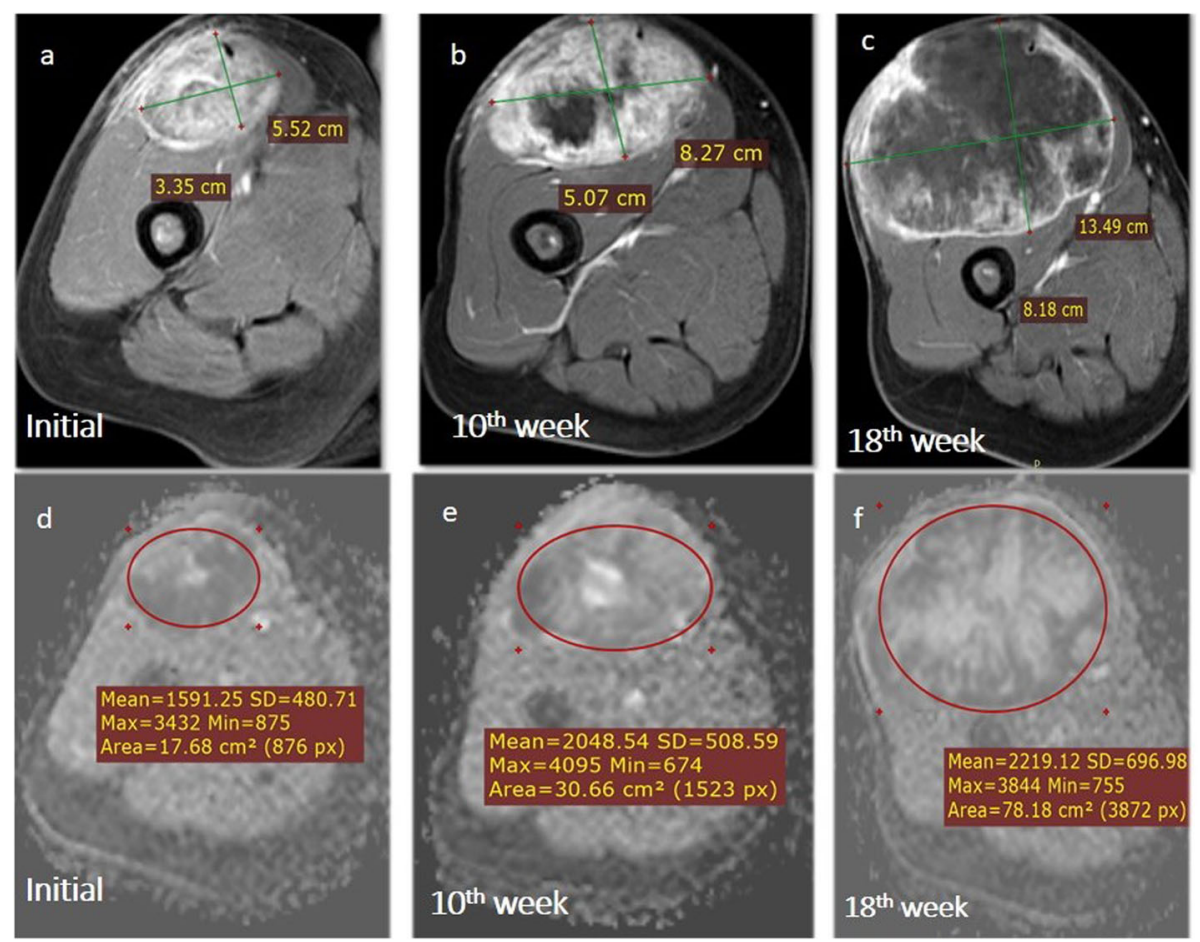

Fig. 1 A 14-year-old male patient with right thigh pleomorphic liposarcoma. Contrast-enhanced T1-weighted images at baseline, 10 and 18 weeks after chemotherapy $(\mathbf{a}-\mathbf{c})$ revealing initially heterogeneous moderately enhancing tumor with size progression and reduction in the tissue contrast enhancement in follow up studies. As compared to the initial MRI study, the tumor showed about (89\%) increase in size classified as PD according to RECIST 1.1; however, it showed (45\%) reduction in size which is classified as PR according to mRECIST. ADC images at baseline, after 10 weeks of chemotherapy and after 18 weeks (d-f) showing an evident increase in ADC values denoting therapeutic response. Wide local excision and the post-operative pathology report showed wide areas of necrosis \& tumor viability is about $40 \%$

- After anatomic imaging, but before any contrast media injection, DWI was performed in the axial plane with multiple b-values; the $b$ values were 0 , 500 , and $1000 \mathrm{~s} / \mathrm{mm}^{2}$.

- ADC maps were calculated from the diffusionweighted images.

- The imaging procedure using the identical scanning protocol was repeated after neoadjuvant therapy.

\section{Imaging evaluation}

- The morphological features of each lesion were recorded including tumor longest dimension according to response evaluation criteria in solid tumors 1.1 (RECIST 1.1), the longest dimension of the contrast-enhanced portion of the tumor according to modified response evaluation criteria in solid tumors (mRECIST), and the tumor volume (VOL) expressed in cubic centimeters $\left(\mathrm{cm}^{3}\right)$ was assessed according to the following equation: $\mathrm{VOL}=$ $(X \times Y \times Z) \times F$, where $\mathrm{X}, \mathrm{Y}$, and $\mathrm{Z}$ represents the longest (transverse, anteroposterior, and cardio-caudal) dimensions respectively, $F=\pi / 4$ in cylindrical lesions and $\pi / 6$ in ellipsoid lesions.
- Signal characteristics and pattern of enhancement of the lesions have been assessed as well.

- Finally, two expert radiologist of 5-10 years' experience reviewed the diffusion images with ADC values in qualitative and quantitative methods with recording ADC values pre- and post-treatment and conjoint results recorded.

\section{ADC calculation}

- The section with the largest tumor diameter was selected for ADC calculation including the visibly most restricted diffusion area on the ADC map, with the region of interest (ROI) being large as possible. Measurements were recorded as a representative value for each case. Initial and follow-up images were matched and ADC calculations were performed on corresponding sections on follow-up MRI.

\section{Interpretation of diffusion-weighted images}

- The lesion was determined on DWI and ADC map by using the conventional MR images as a guide. 

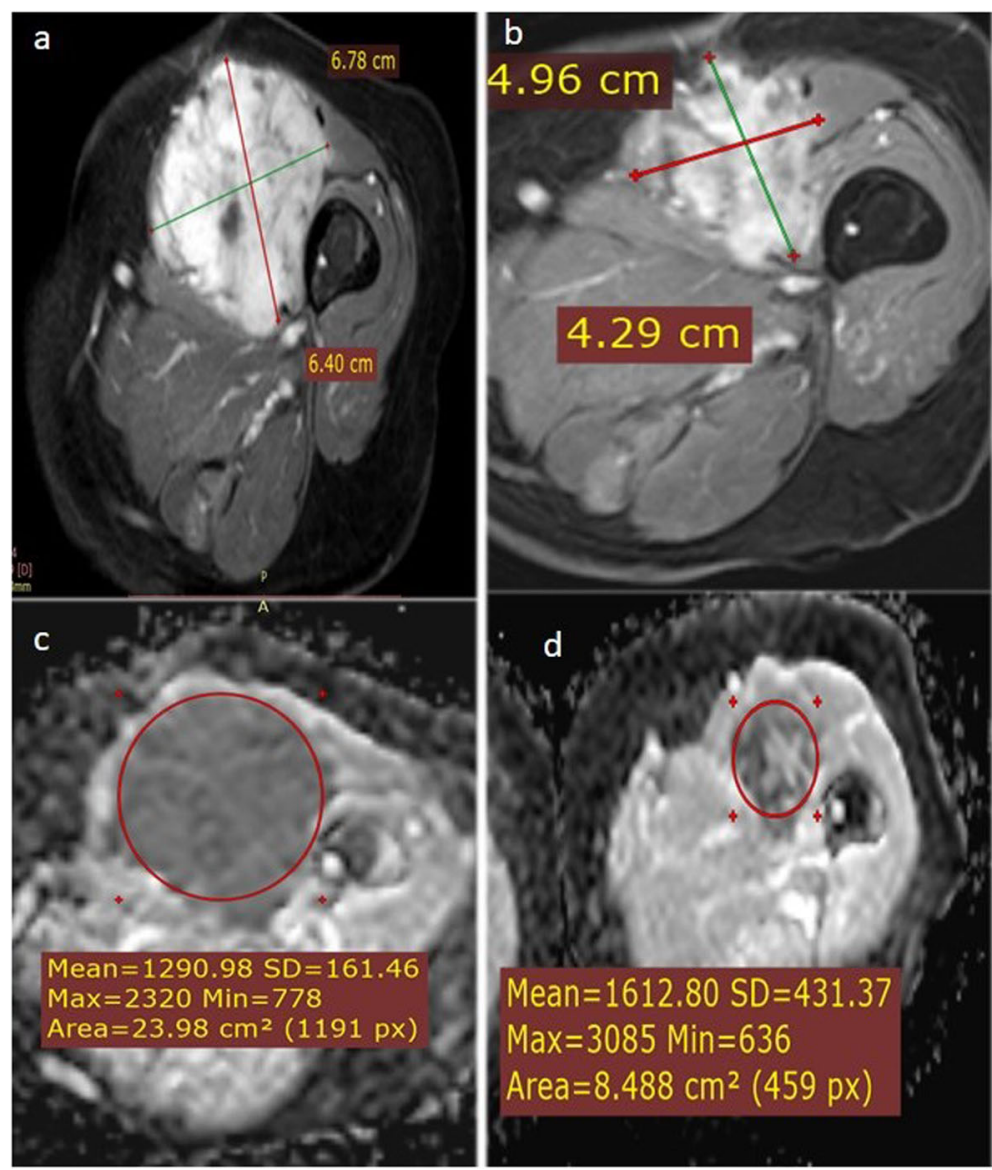

Fig. 2 A 60-year-old female patient, presenting with recurrent left thigh malignant peripheral nerve sheath tumor (MPNST). Contrast-enhanced T1-weighted images at baseline (a) reveal a strongly and almost homogeneously enhancing mass with follow up images after chemotherapy (b) shows a mild reduction of tumor size and contrast enhancement. The tumor showed matched post-treatment response according to both RECIST and mRECIST. There was about (5\%) decrease in size which is classified as SD. ADC images at baseline (c) and after 3 months of chemotherapy (d) showing increased ADC in response to treatment. Wide local excision of the mass revealed recurrent MPNST with an evident therapy effect

Table 2 Detailed analysis of tumor volumes before and after treatment in the progressive and non-progressive group and both groups together with volume variation \%

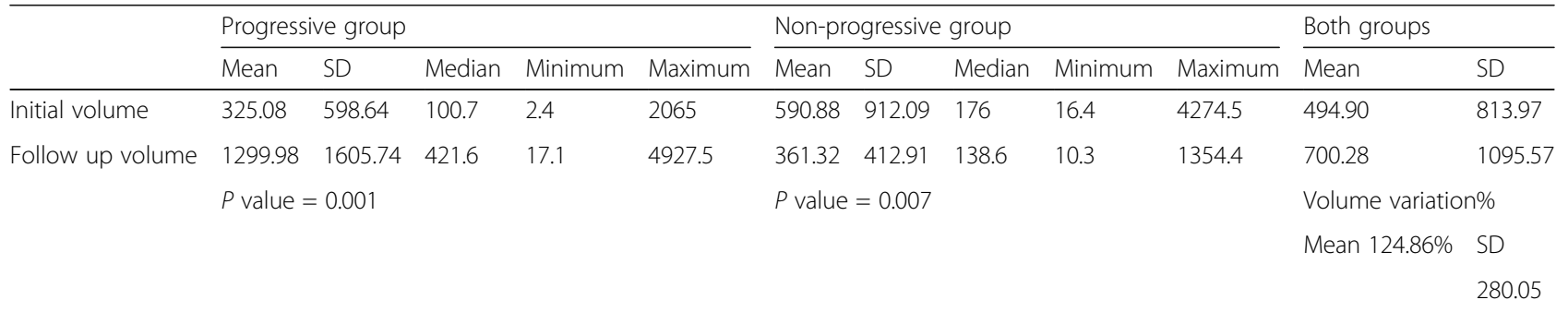


Table 3 Detailed analysis of ADC values among both progressive and non-progressive groups

\begin{tabular}{|c|c|c|c|c|c|c|c|c|c|c|c|}
\hline & \multicolumn{5}{|c|}{ Progressive group } & \multicolumn{5}{|c|}{ Non-progressive group } & \multirow{2}{*}{$\begin{array}{l}P \\
\text { value }\end{array}$} \\
\hline & Mean & SD & Median & Minimum & Maximum & Mean & SD & Median & Minimum & Maximum & \\
\hline ADC initial & 1.33 & 0.38 & 1.20 & 0.80 & 1.90 & 1.35 & 0.41 & 1.30 & 0.60 & 2.20 & 0.820 \\
\hline Follow up ADC & 1.24 & 0.35 & 1.20 & 0.70 & 2.10 & 1.63 & 0.42 & 1.60 & 0.80 & 2.40 & 0.003 \\
\hline ADC\% & -3.08 & 23.5 & -7.00 & -37.00 & 40.00 & 27.09 & 48.09 & 12.00 & -27.00 & 160.00 & 0.043 \\
\hline
\end{tabular}

ADC values after treatment in the non-progressive group were significantly higher than those before treatment $(p<0.05)$. Contrarily, the ADC values after treatment in the progressive group were not significantly different from those before treatment $(p>0.05)$

- The signal intensity of the lesion on DWIs was determined.

- $\mathrm{ADC}$ of the minimum, maximum, and average values was obtained.

\section{Statistical analysis}

1. Statistical analysis was performed using the statistical software: SPSS statistical package version 21 (SPSS Inc., Chicago, IL).

2. MRI features that were analyzed including the location, tumor longest dimension based on (RECIST 1.1) at baseline, and last post neoadjuvant therapy MRI examinations referred to as RECIST 1 and RECIST 2 respectively. Similarly, the longest dimension of the contrast-enhanced portion of the tumor according to (mRECIST) at baseline and last follow up MRI examination referred to as mRECIST 1 and mRECIST 2 respectively, the volume of the tumor before and after neoadjuvant therapy referred to as VOL1 and VOL2 respectively, signal characteristics and enhancement patterns.

3. Differences in tumor volumes (VOL\%) was calculated as $\frac{\text { VOL2-VOL1 }}{\text { VOL1 }} \times 100$, similarly RECIST $\%$ ( $\left.\frac{\text { RECIST 2-RECIST } 1}{\text { RECIST } 1} \times 100\right)$, mRECIST $\%$ $\left(\frac{\mathrm{mRECIST} 2-\mathrm{mRECIST} 1}{\mathrm{mRECIST} 1} \times 100\right)$, and ADC\% $\left(\frac{\mathrm{ADC} 2-\mathrm{ADC} 1}{\mathrm{ADC} 1}\right.$ $\times 100)$ were calculated as well and statistically correlated.

4. The findings on initial MRI were analyzed and correlated with follow up post-treatment MRI examinations and with post-operative histopathological findings when available.

5. ROC curve was constructed with the area under curve analysis performed to detect the best cutoff value of $\mathrm{ADC}$ variations (ADC\%) after neoadjuvant therapy for detection of response to treatment.

6. For comparing categorical data, Chi-square $x^{2}$ test was performed. Exact test was used instead when the expected frequency is less than 5. A $p$ value of less than 0.05 was considered statistically significant.

\section{Results}

Patients' data including histopathological diagnoses, site of primary tumor, treatment plan, and the interval between MRI examinations were demonstrated in Table 1.

\section{Treatment response}

Follow-up was conducted to evaluate neoadjuvant therapy efficacy in accordance with response evaluation criteria in solid tumors (RECIST 1.1). Out of (36) patients, $13(36.1 \%)$ patients had disease progression (PD) (increase in maximum tumor diameter $>20 \%), 12(33.3 \%)$ patients showed stable disease (SD) (change in maximum tumor diameter between PR and progressive disease), and 11 (30.6\%) patients had partial response (PR) (the decrease in maximum tumor diameter $>30$ ) (Fig. 1).

For statistical analysis, the patients were distributed into two groups: the PD group includes 13/36 of patients (36.1\%) and the non-PD group including (PR + SD) 23/ 36 of patients $(63.9 \%)$, according to the detection results (Fig. 2).

\section{MRI imaging features}

Variations in tumor volumes and ADC values among both progressive and non-progressive groups are demonstrated in Tables 2 and 3, respectively.

Correlation between ADC values and tumor volumes before and after neoadjuvant treatment is demonstrated in Table 4. The ADC values after neoadjuvant treatment were negatively related to tumor volumes variations (VOL\%) after neoadjuvant treatment $(p<0.005)$. On the contrary, the ADC values after neoadjuvant treatment were not significantly related to the volumes of the

Table 4 Correlation between VOL\% and ADC after neoadjuvant therapy

\begin{tabular}{lll}
\hline & & VOL\% \\
\hline Follow up ADC values & Correlation coefficient & -0.468 \\
& $P$ value & .004 \\
& $N$ & 36 \\
\hline
\end{tabular}



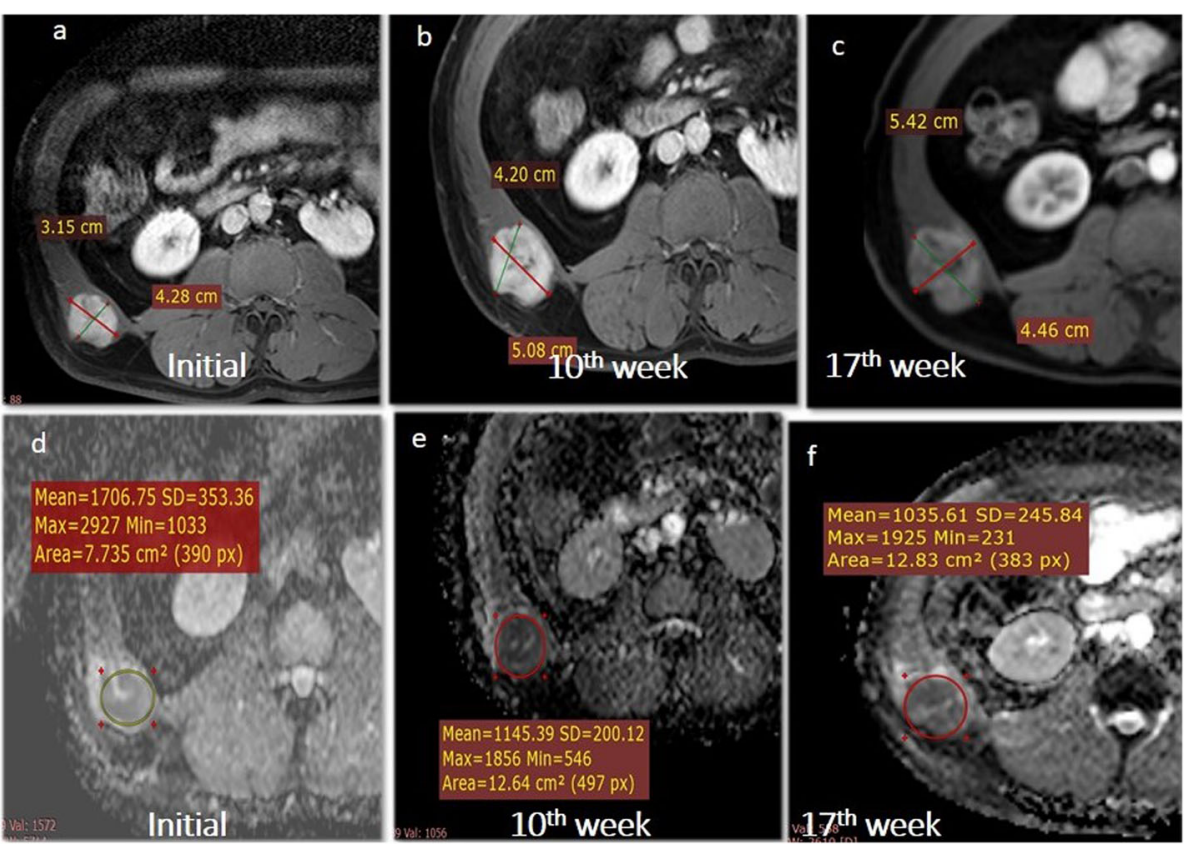

Fig. 3 A 50-year-old male patient presenting with right lumbar pleomorphic RMS. Contrast-enhanced T1-weighted images at baseline, 10 and 17 weeks after chemotherapy $(\mathbf{a}-\mathbf{c})$ revealing heterogeneous moderately enhancing mass with size progression in follow up exams. There was about (35\%) increase in size as compared to the initial study which is classified as PD according to RECIST and m RECIST. ADC images at baseline, after 10 weeks of chemotherapy and after 17 weeks ( $\mathbf{d}-\mathbf{f}$ ) showing decrease of the mean ADC value of the tumor along with the first and second follow-ups

tumors neither before nor after anticancer therapy (Figs. 3 and 4).

\section{Radiological Response Evaluation}

A. Based on RECIST 1.1 (the change in tumor longest dimension) (Tables 5 and 6):

- A decrease in tumor size based on RECIST is associated with an increase in ADC values after neoadjuvant therapy and vice versa. The Pearson correlation coefficient of tumor volumes and ADC values variations was $r=-0.424(p<0.05)$.

B. Based on mRECIST:

- A decrease in tumor size based on mRECIST is associated with an increase in ADC value after neoadjuvant therapy and vice versa. The Pearson correlation coefficient of differences in tumor sizes and ADC values was $r=-0.47(p<0.005)$.

C. Based on tumor volume (VOL):

- A decrease in tumor volume is associated with an increase in ADC value and vice versa. The Pearson correlation coefficient of differences in tumor volumes and ADC values was $r=-0.479(p<0.005)$.
Hence, differences in tumor responses as assessed by RECIST, mRECIST, and 3D volumetric measurements were directly correlated to each other, regardless of the effectiveness of anticancer therapy (Table 7).

ROC curves showed that the areas under the curve (AUC) of the change of ADC values (ADC\%) after neoadjuvant treatment was 0.706 ; the mean percentage of $\mathrm{ADC} \%$ cut-off to differentiate PD and non-PD was $2 \%$ (sensitivity $61.5 \%$ and specificity $78.3 \%$ ) (Table 8 ).

\section{Discussion}

With the advancement of new lines of malignancy treatment alternatives, different imaging modalities are rising, joined by new procedures and rules to survey tumor status and to foresee reaction to chemotherapy [8]. During the treatment plan, it is imperative to evaluate early tumor reaction, so treatment regimens can be suitably custom-made to get greatest tumor reaction [9].

In the study directed by Soldatos et al. [10] that included 23 patients who inferred that expansion of functional sequences to the conventional MR imaging protocol may build affectability for assurance of treatment reaction in soft tissue sarcomas treated preoperatively with neoadjuvant treatment. A number of preclinical and clinical studies have noted that pre-therapy ADC values may indicate therapy outcomes, with most studies showing that tumors with higher ADC values respond less favorably to treatments [11-13]. 

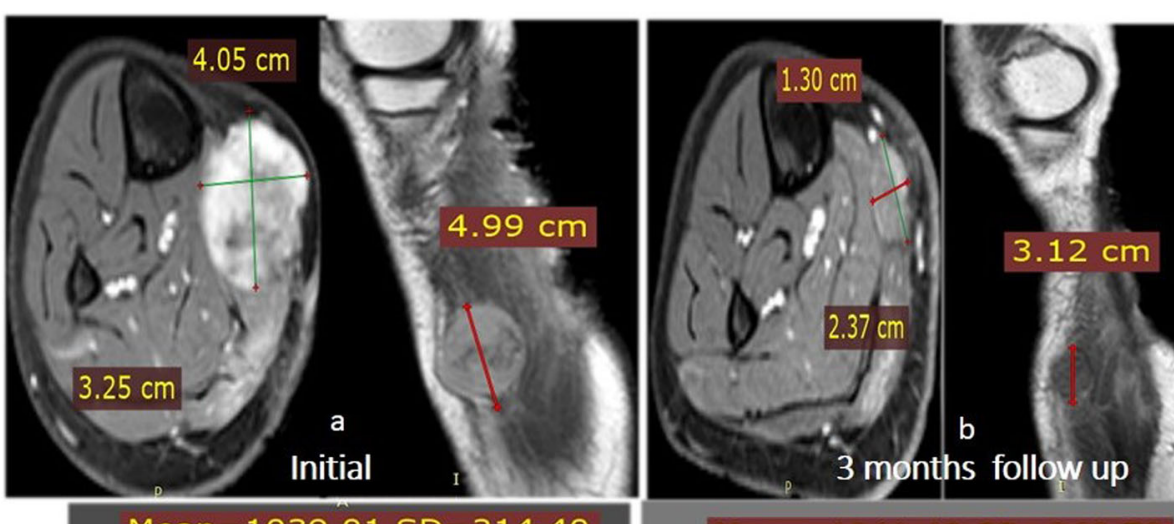

Mean $=1039.01$ SD $=214.40$

$\operatorname{Max}=2258$ Min $=0$

Area $=8.66 \mathrm{~cm}^{2}(429 \mathrm{px})$
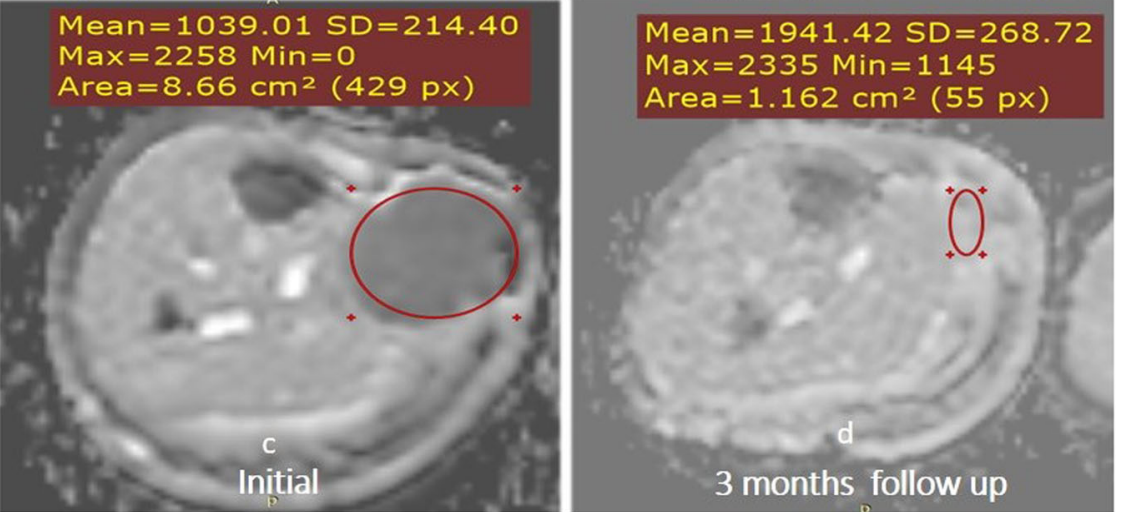

Fig. 4 A 15-year-old male patient, presenting with right leg recurrent undifferentiated spindle cell sarcoma. Contrast-enhanced T1-weighted images at baseline (a) revealing a strongly and almost homogeneously enhancing tumor in the posterior compartment of the right leg with the 3 months follow up images (b) after CCRTH shows marked reduction of tumor size and contrast enhancement. The tumor showed about (36\%) decrease in size which is classified as PR according to RECIST 1.1 and no residual enhancement classified as CR according to $m$ RECIST. ADC images at baseline and after 3 months of CCRT $(\mathbf{c}, \mathbf{d})$ showing facilitated diffusion and increased mean ADC value. Wide local excision of the mass showed no evidence of malignancy denoting good therapeutic response matching both $m$ RECIST and ADC

Contrarily, our study showed that the pre-therapy ADC values in the progressive group showed no difference from those in the non-progressive group.

In support of our results, DeVries et al. [14] highlighted the potential pitfall of using mean tumor ADC values for prognostication in 34 rectal cancer patients undergoing chemoradiation. They showed no differences between mean pretreatment ADC in the 18 patients who responded and the 16 patients who were non-responders. The association between high ADC values and less favorable responses to treatment may not apply to all therapy types [13].

In our study, the ADC values after neoadjuvant treatment in the non-progressive group were significantly higher than those before neoadjuvant treatment (1.63 \pm 0.42 vs. $1.35 \pm 0.41)$ with $(p<0.05)$. However, the ADC values after neoadjuvant treatment in the progressive group were not significantly different from those before neoadjuvant treatment.

That is comparable to the work of Wang et al. [15] who investigated the role of DWI in monitoring the therapeutic response after neoadjuvant chemotherapy in osteosarcoma of long bones in 34 patients; they found that in patients with good response, the post-neoadjuvant chemotherapy values were significantly higher than the pre-neoadjuvant chemotherapy values.

The ADC values after neoadjuvant treatment in our series were negatively related to tumor volumes variations (VOL\%) after neoadjuvant treatment, i.e., tumors that increased in size showed lower ADC values than the tumors that decreased in size after neoadjuvant therapy with the Pearson correlation coefficient $r=-0.46$ $(p<0.005)$.

In our series, the ADC values in the non-progressive group were significantly higher than those of the progressive group after neoadjuvant treatment $(1.63 \pm 0.42$ vs. $1.24 \pm 0.35)$ with $(p<0.005)$. That is comparable to the work of Wang et al. [15] who found that the postneoadjuvant chemotherapy ADC value in patients with a good response was higher than that of poor response.

In our study, $\mathrm{ADC}$ variations (ADC\%) in the nonprogressive group were significantly higher than those of the progressive group $(27.09 \pm 48.09$ vs. $-3.08 \pm 23.5) \%$ with $(p<0.05)$. 


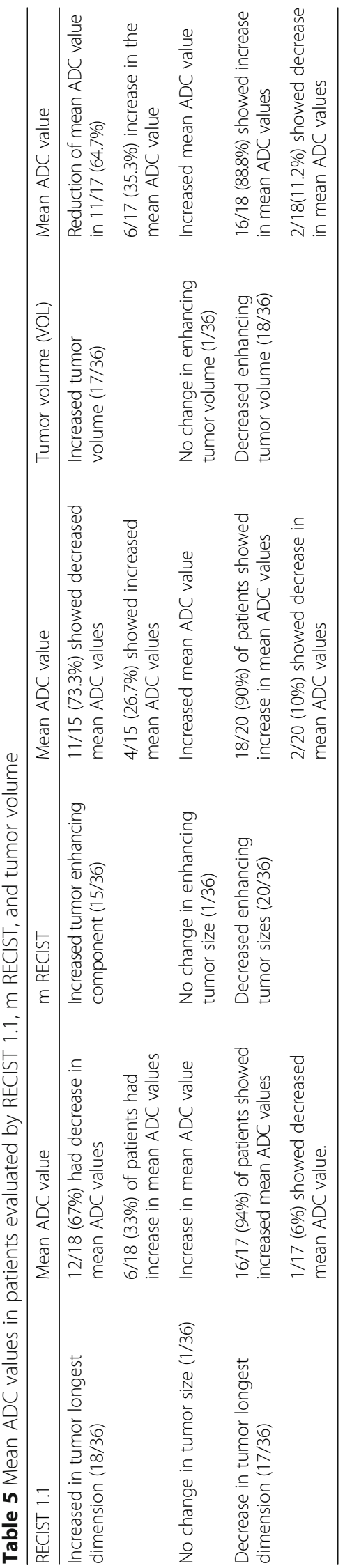


Table 6 Correlation between the different radiological evaluation percentage of change and ADC percentage of change

\begin{tabular}{lll}
\hline & & ADC\% \\
\hline RECIST\% & Correlation Coefficient r $=$ & -0.424 \\
& $P$ value & 0.010 \\
& $N$ & 36 \\
Modified RECIST \% & Correlation Coefficient & -0.478 \\
& P value & 0.003 \\
VOL\% & $N$ & 36 \\
& Correlation Coefficient & -0.479 \\
& $P$ value & 0.003 \\
& $N$ & 36 \\
\hline
\end{tabular}

Our results were supported by the work of Baunin et al. [16] on patients diagnosed with osteosarcoma and found that good responders had a significantly higher $\mathrm{ADC}$ variation $(\mathrm{ADC} \%)$ than poor responders (38.3 \pm 15.09 vs. $12.02 \pm 22.9$ ). However, the ADC differential (ADC2$\mathrm{ADC} 1$ ) of the tumor was also calculated in these cases.

The comparison of ADC results between different series expressed as absolute ADC values still remain difficult. One way to standardize the results is to use ADC differentials (ADC2-ADC1) or variations (ADC\%). ADC variations $(\mathrm{ADC} \%)$ in percentage terms should be more reproducible and could also be more easily understood by clinicians for comparison to histological analysis [16].

In our series, $\mathrm{ADC}$ variations (ADC\%) were inversely correlated with morphologic changes, regardless of the effectiveness of anticancer therapy expressed as changes of tumor size based on (RECIST, mRECIST, and threedimensional volumetric assessment). Linear regression analysis revealed a Pearson correlation coefficient of $r=$ $(-0.424,-0.478$, and -0.479$)$ respectively with $(p<$ 0.005). This relationship was independent of the neoadjuvant therapy protocol or length of the treatment period, with the shortest imaging interval being 30 days, although treatment regimens and imaging intervals were too heterogeneous for statistical analysis.

This is comparable to the study done by Dudeck et al. [17] in which variations in ADC (ADC\%) were inversely correlated with changes of tumor volumes (VOL\%) with a Pearson correlation coefficient of $r=(-0.925)$ and $(p<$ 0.0001). Unlike Dudeck et al.'s study [17], our study revealed that an increase in the ADC value was not always

Table 7 Correlation between variations in tumor sizes based on RECIST, MRECIST, and VOL

\begin{tabular}{llll}
\hline & & VOL\% & Modified RECIST \% \\
\hline RECIST \% & Correlation coefficient & .928 & .741 \\
& $P$ value & $<0.001$ & $<0.001$ \\
& $N$ & 36 & 36 \\
\hline
\end{tabular}

Table 8 ADC variation values (ADC\%) in detecting the efficacy of neoadjuvant treatment in STS

\begin{tabular}{|c|c|c|c|c|c|c|}
\hline \multirow{2}{*}{$\begin{array}{l}\text { Area } \\
\text { under } \\
\text { curve }\end{array}$} & \multirow{2}{*}{$\begin{array}{l}P \\
\text { value }\end{array}$} & \multicolumn{2}{|c|}{ 95\% Confidence interval } & \multirow{2}{*}{$\begin{array}{l}\text { Cutoff } \\
\text { value }\end{array}$} & \multirow[t]{2}{*}{ Sensitivity } & \multirow[t]{2}{*}{ Specificity } \\
\hline & & $\begin{array}{l}\text { Lower } \\
\text { bound }\end{array}$ & $\begin{array}{l}\text { Upper } \\
\text { bound }\end{array}$ & & & \\
\hline 0.706 & 0.043 & 0.531 & 0.880 & 2 & $61.5 \%$ & $78.3 \%$ \\
\hline
\end{tabular}

associated with a reduction of tumor volume. Likewise, a decrease in ADC was not always associated with an increase in tumor volume in all patients.

Based on RECIST 1.1, out of the 23 patients who showed increased ADC values after neoadjuvant therapy in our series: In $16 / 23(63.5 \%)$ of patients, there was decrease in tumor sizes while in $6 / 23(26 \%)$ of patients, there was increase in tumor sizes and $1 / 23$ of patients showed almost no changes in tumor size.

Based on mRECIST, we found that $4 / 6$ of the patients, who showed increased tumor sizes despite increased ADC values, showed the decreased size of the contrastenhanced portion of the tumor. The other two patients showed the increased size of the contrast-enhanced portion with increased cystic components of the tumor.

Similarly, out of the (13) patients who showed decreased ADC values, 12/13 (92.3\%) of patients showed increase in tumor sizes and only one patient showed decrease in tumor size (according to RECIST 1.1); this one patient still showed decreased size according to mRECIST, and this patient was pathologically proven case of extraskeletal Ewing sarcoma who received concomitant chemo-radiotherapy (CCRTH) in the treatment protocol.

In support to our work, Stacchiotti et al. [18] and Taieb et al. [19] stated that in soft-tissue tumors, the effect of targeted therapies can result in different modifications compared with standard cytotoxic chemotherapy.

In an attempt to standardize magnetic resonance imaging techniques and interpretation after neoadjuvant radiotherapy for routine use and within clinical trials, Wardelmann et al. [20] stated that internal signal/density characteristics should be used in combination to assess response. For example, diminished enhancement and reduction in the size of restricted components/rising ADC on DWI may be interpreted as a response.

Our study showed that, according to RECIST 1.1, the disease control rate (defined as the percentage of $\mathrm{CR}+$ PR + SD patients) was $63.8 \%$ (23/36); however, according to mRECIST, the disease control rate was $69.4 \%$ (25/ 36). Evaluation of response to treatment by RECIST1.1 and mRECIST corresponded in $25 / 36$ patients (69.4\%) (PR/SD/PD: 4/11/10, respectively). Three patients who were evaluated as a progressive disease by RECIST1.1 were evaluated by mRECIST is a non-progressive disease (two patient's response changed from $\mathrm{PD}$ to $\mathrm{PR}$, one from $\mathrm{PD}$ to $\mathrm{SD})$. 
Our study had limitations namely the small sample size and heterogeneous group of patients with different sarcoma subtypes.

\section{Conclusion}

Quantitative DW imaging after neoadjuvant therapy provides added value in determining treatment response in soft tissue sarcomas. Therapeutic response to neoadjuvant therapy can be underestimated using RECIST 1.1; therefore, the mRECIST should also be considered.

\section{Abbreviations}

ADC: Apparent diffusion coefficient; CCRTH: Concomitant chemoradiotherapy; DWI: Diffusion-weighted imaging; mRECIST: Modified response evaluation criteria in solid tumors; MRI: Magnetic resonance imaging; PD: Progressive disease; PR: Partial response; RECIST 1.1: Response evaluation criteria in solid tumors 1.1; SD: Stable disease; STS: Soft tissue sarcomas; VOL: Volume

\section{Acknowledgments}

No acknowledgements.

\section{Authors' contributions}

AM wrote the manuscript and is responsible for correspondence to journal. SS collected patient data, image processing, and collection of patients images. MM participated in the design of the study and performed the statistical analysis. $\mathrm{MH}$ conceived of the study, and participated in its design and coordination and helped to draft the manuscript. AS was responsible of revision of the draft from clinical point of view. $\mathrm{MH}$ for oncologic data collection. All authors read and approved the final manuscript.

\section{Funding}

No funding sources.

\section{Availability of data and materials}

The datasets used and analyzed during the current study are available from the corresponding author on reasonable request.

\section{Ethics approval and consent to participate}

The study was approved by the ethical committee of the faculty of medicine, Cairo University on October 2017 with ID number 51/2017 and an informed written consent was taken from all subjects.

\section{Consent for publication}

All patients included in this research gave written informed consent to publish the data contained within this study.

\section{Competing interests}

The authors declare that they have no competing interests.

\section{Author details}

${ }^{1}$ Department of Diagnostic and Interventional Radiology, National cancer institute, Cairo University, 1 kasr Elainy street Fom Elkalig, Cairo, Egypt. ${ }^{2}$ Department of Diagnostic and Interventional Radiology, Faculty of Medicine, Cairo University, Cairo, Egypt. ${ }^{3}$ Department of Medical Oncology, National Cancer Institute, Cairo University, Cairo, Egypt.

Received: 13 August 2019 Accepted: 1 October 2019 Published online: 21 November 2019

\section{References}

1. Jo WY, Fletcher CDM (2014) WHO classification of soft tissue tumours: an update based on the 2013 (4th) edition. Pathology 46:95-104. https://doi. org/10.1097/PAT.0000000000000050

2. Loong HH, Wong K-H, Tse T (2018) Controversies and consensus of neoadjuvant chemotherapy in soft-tissue sarcomas. ESMO open 3:e000293. https://doi.org/10.1136/esmoopen-2017-000293
3. von Mehren M, Randall RL, Benjamin RS, Boles S, Bui MM, Ganjoo KN, George S, Gonzalez RJ, Heslin MJ, Kane JM, Keedy V, Kim E, Koon H, Mayerson J, McCarter M, McGarry SV, Meyer C, Morris ZS, O'Donnell RJ, Pappo AS, Paz IB, Petersen IA, Pfeifer JD, Riedel RF, Ruo B, Schuetze S, Tap WD, Wayne JD, Bergman MA, Scavone JL (2018) Soft Tissue Sarcoma, Version 2.2018, NCCN Clinical Practice Guidelines in Oncology. J Natl Compr Cancer Netw J Natl Compr Canc Netw 16:536-563. https://doi.org/10.6004/ jncen.2018.0025

4. Al-Absi E, Farrokhyar F, Sharma R, Whelan K, Corbett T, Patel M, Ghert M (2010) A systematic review and meta-analysis of oncologic outcomes of pre- versus postoperative radiation in localized resectable soft-tissue sarcoma. Ann Surg Oncol 17:1367-1374. https://doi.org/10.1245/s10434009-0885-7

5. Dancsok AR, Asleh-Aburaya K, Nielsen TO (2017) Advances in sarcoma diagnostics and treatment. Oncotarget 8:7068-7093. https://doi.org/10. 18632/oncotarget.12548

6. Subhawong TK, Jacobs MA, Fayad LM (2014) Diffusion-weighted MR imaging for characterizing musculoskeletal lesions. RadioGraphics 34: 1163-1177. https://doi.org/10.1148/rg.345140190

7. Subhawong TK, Durand DJ, Thawait GK, Jacobs MA, Fayad LM (2013) Characterization of soft tissue masses: can quantitative diffusion weighted imaging reliably distinguish cysts from solid masses? Skeletal Radiol 42: 1583-1592. https://doi.org/10.1007/s00256-013-1703-7

8. Kang H, Lee HY, Lee KS, Kim J-H (2012) Imaging-based tumor treatment response evaluation: review of conventional, new, and emerging concepts. Korean J Radiol 13:371. https://doi.org/10.3348/kjr.2012.13.4.371

9. Rastogi P, Anderson SJ, Bear HD, Geyer CE, Kahlenberg MS, Robidoux A, Margolese RG, Hoehn JL, Vogel VG, Dakhil SR, Tamkus D, King KM, Pajon ER, Wright MJ, Robert J, Paik S, Mamounas EP, Wolmark N (2008) Preoperative chemotherapy: updates of national surgical adjuvant breast and bowel project protocols B-18 and B-27. J Clin Oncol 26:778-785. https://doi.org/10. 1200/JCO.2007.15.0235

10. Soldatos T, Ahlawat S, Montgomery E, Chalian M, Jacobs MA, Fayad LM (2016) Multiparametric assessment of treatment response in high-grade soft-tissue sarcomas with anatomic and functional MR imaging sequences. Radiology 278:831-840. https://doi.org/10.1148/radiol.2015142463

11. Thoeny HC, Ross BD (2010) Predicting and monitoring cancer treatment response with diffusion-weighted MRI. J Magn Reson Imaging 32:2-16. https://doi.org/10.1002/jmri.22167

12. Cui $Y$, Zhang X-P, Sun Y-S, Tang $L$, Shen $L$ (2008) Apparent diffusion coefficient: potential imaging biomarker for prediction and early detection of response to chemotherapy in hepatic metastases. Radiology 248:894900. https://doi.org/10.1148/radiol.2483071407

13. Li SP, Padhani AR (2012) Tumor response assessments with diffusion and perfusion MRI. J Magn Reson Imaging 35:745-763. https://doi.org/10.1002/ jmri.22838

14. DeVries AF, Kremser C, Hein PA, Griebel J, Krezcy A, Ofner D, Pfeiffer KP, Lukas P, Judmaier W (2003) Tumor microcirculation and diffusion predict therapy outcome for primary rectal carcinoma. Int J Radiat Oncol Biol Phys 56:958-965. https://doi.org/10.1016/s0360-3016(03)00208-6

15. Wang C-S, Du L-J, Si M-J, Yin Q-H, Chen L, Shu M, Yuan F, Fei X-C, Ding X-Y (2013) Noninvasive assessment of response to neoadjuvant chemotherapy in osteosarcoma of long bones with diffusion-weighted imaging: an initial in vivo study. PLoS One 8:e72679. https://doi.org/10.1371/journal.pone.0072679

16. Baunin C, Schmidt G, Baumstarck K, Bouvier C, Gentet JC, Aschero A, Ruocco A, Bourlière B, Gorincour G, Desvignes C, Colavolpe N, Bollini G, Auqier P, Petit P (2012) Value of diffusion-weighted images in differentiating mid-course responders to chemotherapy for osteosarcoma compared to the histological response: preliminary results. Skeletal Radiol 41:1141-1149. https://doi.org/10.1007/s00256-012-1360-2

17. Dudeck O, Zeile M, Pink D, Pech M, Tunn P-U, Reichardt P, Ludwig W-D, Hamm B (2008) Diffusion-weighted magnetic resonance imaging allows monitoring of anticancer treatment effects in patients with soft-tissue sarcomas. J Magn Reson Imaging 27:1109-1113. https://doi.org/10.1002/jmri.21358

18. Stacchiotti S, Negri T, Libertini M, Palassini E, Marrari A, De Troia B, Gronchi A, Dei Tos AP, Morosi C, Messina A, Pilotti S, Casali PG (2012) Sunitinib malate in solitary fibrous tumor (SFT). Ann Oncol 23:3171-3179. https://doi. org/10.1093/annonc/mds143

19. Taieb S, Saada-Bouzid E, Tresch E, Ryckewaert T, Bompas E, Italiano A, Guillemet C, Peugniez C, Piperno-Neumann S, Thyss A, Maynou C, Clisant S, Penel N, French Sarcoma Group (2015) Comparison of response evaluation 
criteria in solid tumours and choi criteria for response evaluation in patients with advanced soft tissue sarcoma treated with trabectedin: A retrospective analysis. Eur J Cancer 51:202-209. https://doi.org/10.1016/j.ejca.2014.11.008

20. Wardelmann E, Schildhaus H-U, Merkelbach-Bruse S, Hartmann W, Reichardt

P, Hohenberger P, Büttner R (2010) Soft tissue sarcoma: from molecular diagnosis to selection of treatment. Pathological diagnosis of soft tissue sarcoma amid molecular biology and targeted therapies. Ann Oncol 21: vii265-vii269. https://doi.org/10.1093/annonc/mdq381

\section{Publisher's Note}

Springer Nature remains neutral with regard to jurisdictional claims in published maps and institutional affiliations.

Submit your manuscript to a SpringerOpen ${ }^{\circ}$ journal and benefit from:

- Convenient online submission

Rigorous peer review

- Open access: articles freely available online

High visibility within the field

- Retaining the copyright to your article

Submit your next manuscript at $\boldsymbol{\nabla}$ springeropen.com 